\title{
Publisher Correction: Precise targeting of POLR2A as a therapeutic strategy for human triple negative breast cancer
}

Jiangsheng Xu, Yunhua Liu, Yujing Li, Hai Wang, Samantha Stewart, Kevin Van der Jeught D, Pranay Agarwal, Yuntian Zhang, Sheng Liu, Gang Zhao DiD, Jun Wan, Xiongbin Lu and Xiaoming He (D)

Correction to: Nature Nanotechnology https://doi.org/10.1038/s41565-019-0381-6, published online 25 February 2019.

The Supplementary Information originally published with this Article was an older version, in which 'IFN- $\gamma$ ' was misspelled 'INF- $\gamma$ ' in Supplementary Fig. 9 and the $\beta$-Actin blot in Supplementary Fig. 13 was the wrong image. The Supplementary Information has now been replaced.

Published online: 5 March 2019

https://doi.org/10.1038/s41565-019-0417-y

\section{Publisher Correction: Micelles from gemini}

Alberto Moscatelli

Correction to: Nature Nanotechnology https://doi.org/10.1038/s41565-019-0431-0, published online 2 April 2019.

In the version of this Research Highlight originally published, the text 'Now, Nishioka et al. have shown that carefully designed gemini amphiphiles can also form micelles' should have read 'Now, Nishioka et al. have shown that aromatic gemini amphiphiles can form monodisperse micelles'. This has now been corrected.

Published online: 10 April 2019

https://doi.org/10.1038/s41565-019-0451-9 